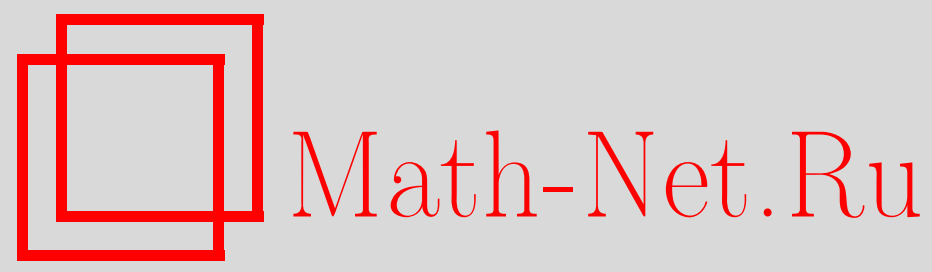

Л. А. Масальцев, Непогружаемость нилмногообразий в виде гиперповерхностей в евклидово пространство, Матем. заметки, 2004, том 76, выпуск 6, 868-873

DOI: https://doi.org/10.4213/mzm158

Использование Общероссийского математического портала Math-Net.Ru подразумевает, что вы прочитали и согласны с пользовательским соглашением http://www.mathnet.ru/rus/agreement

Параметры загрузки:

IP : 3.85 .5 .30

26 апреля 2023 г., 09:43:53

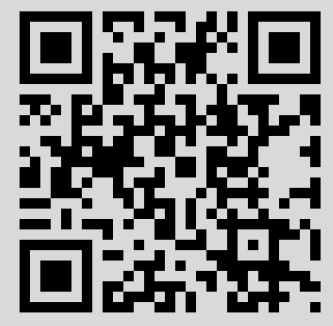




\section{НЕПОГРУЖАЕМОСТЬ НИЛМНОГООБРАЗИЙ В ВИДЕ ГИПЕРПОВЕРХНОСТЕЙ В ЕВКЛИДОВО ПРОСТРАНСТВО}

\section{Л.А. Масальцев}

Доказано, что $2 n+1$-мерная группа Гейзенберга $H_{n}$ и 4 -многообразия $\mathrm{Nil}^{4}, \mathrm{Nil}^{3} \times \mathbb{R}$ c произвольной левоинвариантной метрикой не допускают $C^{3}$-регулярного погружения в виде гиперповерхностей в евклидовы пространства $\mathbb{R}^{2 n+2}$ и $\mathbb{R}^{5}$ соответственно.

Библиография: 7 названий.

В работе [1] Ривертц доказал теорему о том, что не существует регулярного изометрического погружения трехмерной грушы Гейзенберга с произвольной левоинвариантной метрикой в евклидово пространство $\mathbb{R}^{4}$. Напомним, что дифференцируемое отображение $f: M^{m} \rightarrow N^{n}$ дифференцируемых многообразий назьвается погружсением, если его дифференциал $f_{\star}(x): T_{x} M \rightarrow T_{f(x)} N$ является инъективньм для всех $x \in M^{m}$. Погружение риманова многообразия $M^{m}$ с метрикой $\langle\cdot, \cdot\rangle_{M}$ в риманово многообразие $N^{n}$ с метрикой $\langle\cdot, \cdot\rangle_{N}$ называется изометрическим, если $\langle X, Y\rangle_{M}=\left\langle f_{\star} X, f_{\star} Y\right\rangle_{N}$ для всех $x \in M$ и всех $X, Y \in T_{x} M$. Обзор результатов по изометрическим погружениям с доказательствами содержится в главе 3 монографии [2] Громова.

В настоящей заметке мы обобщаем результат Ривертца на $(2 n+1)$-мерную группу Гейзенберга $H_{n}$ с произвольной левоинвариантной метрикой.

ТЕорема 1. Не существует $C^{3}$-регулярного изометрического погружения $(2 n+1)$-мерной группы Гейзенберга $H_{n}$ в евклидово пространство $\mathbb{R}^{2 n+2}$.

Известно [3, п. 7.3], что существует три односвязных 4-мерных нильпотентных группы Ли: $\mathbb{R}^{4}, \mathrm{Nil}^{3} \times \mathbb{R}, \mathrm{Nil}^{4}$. Для них мы получаем следующий результат.

ТЕорема 2. Не существует $C^{3}$-регулярного изометрического погружения 4мерных групп $\mathrm{Nil}^{3} \times \mathbb{R}, \mathrm{Nil}^{4}$ с произвольными левоинвариантными метриками в евклидово пространство $\mathbb{R}^{5}$.

Следует заметить, что нилмногообразия в теоремах 1 и 2 допускают гладкие (неизометрические) погружения коразмерности 1 в евклидовы пространства по теореме Смейла-Хирша. Известная теорема Черна-Кейпера [4, с. 34, теорема 4.7] утверждает, что если в каждой точке $x$ компактного риманова многообразия $M^{m}$ касательное пространство $T_{x} M$ содержит $k$-мерное подпространство $T_{x}^{\prime}$ такое, что секционная кривизна любой плоскости из $T_{x}^{\prime}$ неположительна, то коразмерность $p$ изометрического погружения $M^{m}$ в евклидово пространство $\mathbb{R}^{m+p}$ удовлетворяет неравенству $p \geqslant k$. Поскольку у нилмногообразий, рассмотренных в теоремах 1 и 2 , в любой точке $x$ имеются двумерные плоскости в $T_{x} M$, в направлении которых секционная кривизна отрицательна, то для их компактных фактор-многообразий теорема Черна-Кейпера верифицирует 
результаты теорем 1 и 2. Заметим также, что поскольку рассматриваемые нилмногообразия аналитические, то согласно классической теореме Жане [2, с. 25] существует окрестность любой точки $x \in M^{m}$, допускающая изометрическое аналитическое вложение в евклидово пространство $\mathbb{R}^{m(m+1) / 2}$.

1. Риманова группа Гейзенберга $H_{n}$ и ее алгебра Ли. Приведем необходимые сведения, которые содержатся в [5], сохраняя введенные там обозначения. $(2 n+1)$-мерная әруппа Гейзенберга есть подгруппа $H_{n}=\left(h(x, y, t): x, y \in \mathbb{R}^{n}, t \in \mathbb{R}\right)$ групшы $G L(n+2, \mathbb{R})$, где

$$
h(x, y, t)=\left(\begin{array}{ccccc}
1 & x_{1} & \ldots & x_{n} & t \\
0 & 1 & \ldots & 0 & y_{1} \\
\ldots & \ldots & \ldots & \ldots & \ldots \\
0 & 0 & \ldots & 1 & y_{n} \\
0 & 0 & \ldots & 0 & 1
\end{array}\right)
$$

Умножение в группе задается законом

$$
h(x, y, t) h\left(x^{\prime}, y^{\prime}, t^{\prime}\right)=h\left(x+x^{\prime}, y+y^{\prime}, t+t^{\prime}+\left\langle x, y^{\prime}\right\rangle\right)
$$

где $\left\langle x, y^{\prime}\right\rangle$ есть стандартное скалярное произведение в $\mathbb{R}^{n}$. Алгебра Ли $h_{n}$ грушшы $H_{n}$ имеет базис $X_{1}, \ldots, X_{n}, Y_{1}, \ldots, Y_{n}, Z$, образующие которого удовлетворяют соотношениям $\left[X_{i}, Y_{i}\right]=Z, i=1, \ldots, n$, и остальные скобки равны нулю. Всякая левоинвариантная метрика на $H_{n}$ однозначно определяется ассоциированным внутренним произведением на касательном пространстве $T_{e}\left(H_{n}\right)=h_{n}$ в единице $e$. В [5, лемма 2.8] доказано, что всякая левоинвариантная метрика на $H_{n}$ изометрична единственной метрике с внутренним скалярным произведением в $h_{n}$ диагонального вида

$$
\left\langle X_{i}, X_{i}\right\rangle=\left\langle Y_{i}, Y_{i}\right\rangle=a_{i}, \quad\langle Z, Z\rangle=1,
$$

где $0<a_{1} \leqslant \cdots \leqslant a_{n}$.

2. Непогружаемость группы Гейзенберга $H_{n}$ в евклидово пространство $\mathbb{R}^{2 n+2}$. Для доказательства теоремы 1 нам потребуется выгислить компоненты тензора Римана $R_{i, k, i, k}$ и $R_{i, r, i, j}$ левоинвариантной метрики в единище $e \in H_{n}$.

Лемма. Среди компонент вида $R_{i, k, i, k} u R_{i, r, i, j}$ тензора Римана левоинвариантной метрики (1) в единице е ненулевыми являются следующие:

$$
R_{i, n+i, i, n+i}=-\frac{3}{4}, \quad R_{i, 2 n+1, i, 2 n+1}=R_{n+i, 2 n+1, n+i, 2 n+1}=\frac{1}{4 a_{i}}, \quad i=1, \ldots, n .
$$

ДоКАЗАТЕЛЬСТво. Согласно [6, предложение 7.28] для ковариантной производной левоинвариантной метрики в точке $e$ справедлива формула $\left(\nabla_{P} Q\right)_{e}=-\frac{1}{2}[P, Q]+$ $U(P, Q)$, где симметрическое отображение $U: h_{n} \times h_{n} \rightarrow h_{n}$ определяется равенством

$$
2\langle U(P, Q), R\rangle=\langle[R, P], Q\rangle+\langle P,[R, Q]\rangle \quad \text { для всех } P, Q, R \in h_{n} .
$$

Учитьвая выражение левоинвариантной метрики в точке $e$, получим

$$
U\left(X_{i}, Z\right)=-\frac{1}{2 a_{i}} Y_{i}, \quad U\left(Y_{i}, Z\right)=\frac{1}{2 a_{i}} X_{i}, \quad i=1, \ldots, n .
$$


Значения отображения $U$ на остальных парах базисных векторов равны нулю. Для ненулевых ковариантных производных получим следующие выражения:

$$
\begin{gathered}
\nabla_{X_{i}} Y_{i}=-\frac{1}{2} Z, \quad \nabla_{Y_{i}} X_{i}=\frac{1}{2} Z \\
\nabla_{X_{i}} Z=-\frac{1}{2 a_{i}} Y_{i}, \quad \nabla_{Y_{i}} Z=\frac{1}{2 a_{i}} X_{i}, \quad \nabla_{Z} X_{i}=-\frac{1}{2 a_{i}} Y_{i}, \quad \nabla_{Z} Y_{i}=\frac{1}{2 a_{i}} X_{i}, \quad i=1, \ldots, n .
\end{gathered}
$$

Для вьгисления компонент тензора Римана $R_{i, k, i, k}$ можно прямо использовать формулу [6, п. 7.30]

$$
\begin{aligned}
R_{i, k, i, k}=\left\langle R\left(V_{i}, V_{k}\right) V_{i}, V_{k}\right\rangle= & -\frac{3}{4}\left|\left[V_{i}, V_{k}\right]\right|^{2}-\frac{1}{2}\left\langle\left[V_{i},\left[V_{i}, V_{k}\right]\right], V_{k}\right\rangle-\frac{1}{2}\left\langle\left[V_{k},\left[V_{k}, V_{i}\right]\right], V_{i}\right\rangle \\
& +\left|U\left(V_{i}, V_{k}\right)\right|^{2}-\left\langle U\left(V_{i}, V_{i}\right), U\left(V_{k}, V_{k}\right)\right\rangle .
\end{aligned}
$$

Применяя эту формулу, получим следующие ненулевые компоненты (мы предполагаем, что базис алгебры Ли группы $H_{n}$ упорядочен в связи с вьше приведенной формулой следуюшим образом: $V_{1}=X_{1}, \ldots, V_{n}=X_{n}, V_{n+1}=Y_{1}, \ldots, V_{2 n}=Y_{n}, V_{2 n+1}=Z$ )

$$
R_{i, n+i, i, n+i}=-\frac{3}{4}, \quad R_{i, 2 n+1, i, 2 n+1}=R_{n+i, 2 n+1, n+i, 2 n+1}=\frac{1}{4 a_{i}}, \quad i=1, \ldots, n
$$

Вычисление компонент вида $R_{i, k, i, j}=\left\langle R\left(V_{i}, V_{j}\right) V_{k}, V_{i}\right\rangle$ проводится непосредственно с использованием формулы $R\left(V_{i}, V_{j}\right) V_{k}=\nabla_{V_{i}} \nabla_{V_{j}} V_{k}-\nabla_{V_{j}} \nabla_{V_{i}} V_{k}-\nabla_{\left[V_{i}, V_{j}\right]} V_{k}$ и дает нулевые значения для всех них.

ЗАмечАниЕ. Для групшы Гейзенберга $H_{2}$ нетрудно найти следующее выражение левоинвариантной метрики

$$
d s^{2}=a_{1}\left(d x_{1}^{2}+d y_{1}^{2}\right)+a_{2}\left(d x_{2}^{2}+d y_{2}^{2}\right)+\left(x_{1} d y_{1}+x_{2} d y_{2}-d t\right)^{2}
$$

Компютерные вычисления в Maple с использованием опции Riemann в пакете для работы с тензорами with (tensor) дают следующие значения для ненулевых компонент тензора Римана:

$$
\begin{gathered}
R_{1,3,1,3}=R_{2,4,2,4}=-\frac{1}{4 D}\left(-3 a_{1} a_{2}+3 x_{1} x_{2}^{2}-3 x_{1}^{2} x_{2}^{2}+a_{2} x_{1}^{2}+a_{1} x_{2}^{2}\right), \quad R_{2,5,2,5}=-\frac{a_{1}}{4 D} \\
R_{1,5,1,5}=-\frac{a_{2}}{4 D}, \quad R_{4,5,4,5}=\frac{1}{4 a_{2}}, \quad R_{3,5,3,5}=\frac{1}{4 a_{1}}, \quad R_{1,5,2,3}=-R_{1,2,3,5}=\frac{a_{1} x_{2}}{4 D} \\
R_{1,4,2,3}=-R_{1,2,3,4}=-\frac{1}{4 D}\left(x_{1}^{2} x_{2}^{2}-a_{2} x_{1}^{2}-a_{1} x_{2}^{2}+a_{1} a_{2}-x_{1} x_{2}^{2}\right) \\
R_{1,4,2,5}=R_{1,2,4,5}=-R_{1,3,1,5}=-\frac{a_{2} x_{1}}{4 D}, \quad R_{2,4,2,5}=\frac{a_{1} x_{2}}{4 D}
\end{gathered}
$$

где $D=-a_{1} a_{2}+x_{1} x_{2}^{2}-x_{1}^{2} x_{2}^{2}$.

Легко видеть, что в точке $e$, т.е. при $x_{1}=x_{2}=0$ получаются значения компонент, указанные в лемме. 
ДокАЗАТЕЛЬСТво ТЕОРЕмы 1. Допустим, что существует регулярное класса $C^{3}$ погружение римановой грушы Гейзенберга $H_{n}$ (размерности $\left.2 n+1\right)$ в евклидово пространство $\mathbb{R}^{2 n+2}$. Тогда имеет место система уравнений Гаусса в точке $e[7$, c. 66$]$

$$
R_{i, j, k, l}=b_{i k} b_{j l}-b_{i l} b_{j k}, \quad 1 \leqslant i, j, k, l \leqslant 2 n+1
$$

где $R_{i, j, k, l}$ - компоненты тензора Римана групшы $H_{n}$, погруженной в виде гиперповерхности в $\mathbb{R}^{2 n+2}$, и $b_{i k}$ - коэффициенты ее второй фундаментальной формы.

Дальше мы выписьваем не все уравнения Гаусса, а только те, которые потребуются для доказательства противоречивости:

$$
\begin{gathered}
R_{i, n+i, i, n+i}=b_{i i} b_{n+i n+i}-b_{i n+i}^{2}=-\frac{3}{4} \\
R_{i, 2 n+1, i, 2 n+1}=b_{i i} b_{2 n+12 n+1}-b_{i 2 n+1}^{2}=\frac{1}{4 a_{i}}, \quad i=1, \ldots, n \\
R_{n+i, 2 n+1, n+i, 2 n+1}=b_{n+i n+i} b_{2 n+12 n+1}-b_{n+i 2 n+1}^{2}=\frac{1}{4 a_{i}}, \\
R_{k, t, t, s}=b_{k t} b_{t s}-b_{k s} b_{t t}=0
\end{gathered}
$$

где индексы $1 \leqslant k, t, s \leqslant 2 n+1$ попарно разные. Рассмотрим тройку следующих уравнений из данной системы:

$$
\begin{aligned}
R_{i, 2 n+1, i, 2 n+1} & =b_{i i} b_{2 n+12 n+1}-b_{i 2 n+1}^{2}=\frac{1}{4 a_{i}} \\
R_{k, i, i, 2 n+1} & =b_{k i} b_{i 2 n+1}-b_{k 2 n+1} b_{i i}=0 \\
R_{k, 2 n+1,2 n+1, i} & =b_{k 2 n+1} b_{2 n+1 i}-b_{k i} b_{2 n+12 n+1}=0
\end{aligned}
$$

где $1 \leqslant i \leqslant n, 1 \leqslant k \leqslant 2 n, i \neq k$.

Если умножить второе из этих уравнений на $b_{2 n+12 n+1}$, а третье - на $b_{i 2 n+1}$ и почленно сложить, то получим

$$
b_{k 2 n+1}\left(b_{i 2 n+1}^{2}-b_{i i} b_{2 n+12 n+1}\right)=0 .
$$

Откуда, с учетом первого уравнения следует, что $b_{k 2 n+1}=0$. Аналогично, если умножить второе уравнение на $b_{i 2 n+1}$, третье-на $b_{i i}$ и сложить, то с учетом первого получим $b_{k i}=0$. В частности, имеем $b_{12 n+1}=b_{1 n+1}=b_{n+12 n+1}=0$. Тогда тройка уравнений

$$
\begin{gathered}
R_{1, n+1,1, n+1}=b_{11} b_{n+1 n+1}=-\frac{3}{4}, \quad R_{1,2 n+1,1,2 n+1}=b_{11} b_{2 n+12 n+1}=\frac{1}{4 a_{1}} \\
R_{n+1,2 n+1, n+1,2 n+1}=b_{n+1 n+1} b_{2 n+12 n+1}=\frac{1}{4 a_{1}}
\end{gathered}
$$

противоречива, поскольку из них следует, что $b_{11}^{2}<0$, что и доказывает непогружаемость $H_{n}$ в $\mathbb{R}^{2 n+2}$. 
3. $\mathrm{Nil}^{4}$-многообразие и его левоинвариантная метрика. Как известно [3, c. 135], группа $\mathrm{Nil}^{4}$ представляет собой полупрямое произведение $\mathbb{R}^{3} \times_{\theta} \mathbb{R}$, где

$$
\theta(t)=\left(\begin{array}{ccc}
1 & t & \frac{t^{2}}{2} \\
0 & 1 & t \\
0 & 0 & 1
\end{array}\right)
$$

Следовательно, умножение в $\mathrm{Nil}^{4}$ задается законом

$$
(g, s)(h, t)=(g+\theta(s) h, s+t)=\left(\left(\begin{array}{c}
g_{1}+h_{1}+s h_{2}+\frac{S^{2}}{2} h_{3} \\
g_{2}+h_{2}+s h_{3} \\
g_{3}+h_{3}
\end{array}\right), s+t\right),
$$

где $g, h \in \mathbb{R}^{3}, t, s \in \mathbb{R}$. Если метрический тензор левоинвариантной метрики $\mathrm{Nil}^{4}$ в точке $e$ имеет диагональный вид $g_{i j}(e)=\delta_{i j} a_{i}$, где $a_{i}>0, i=1,2,3,4$, то нетрудно найти его выражение в произвольной точке $(g, s)$ :

$$
g_{i j}((g, s))=\left(\begin{array}{cccc}
a_{1} & -a_{1} s & a_{1} \frac{s^{2}}{2} & 0 \\
-a_{1} s & a_{1} s^{2}+a_{2} & -s\left(a_{2}+a_{1} \frac{s^{2}}{2}\right) & 0 \\
a_{1} \frac{s^{2}}{2} & -s\left(a_{2}+a_{1} \frac{s^{2}}{2}\right) & a_{1} \frac{s^{4}}{4}+a_{2} s^{2}+a_{3} & 0 \\
0 & 0 & 0 & a_{4}
\end{array}\right) .
$$

Ненулевые компоненты тензора Римана в точке $e$, вычисленные в Maple, с использованием опции Riemann в пакете with (tensor), имеют следующие значения:

$$
\begin{gathered}
R_{2,3,2,3}=\frac{a_{2}^{2}}{4 a_{4}}, \quad R_{1,4,3,4}=-\frac{a_{1}}{4}, \quad R_{3,4,3,4}=-\frac{3 a_{2}}{4}, \quad R_{1,4,1,4}=\frac{a_{1}^{2}}{4 a_{2}}, \\
R_{1,2,2,3}=-\frac{a_{1} a_{2}}{4 a_{4}}, \quad R_{1,2,1,2}=\frac{a_{1}^{2}}{4 a_{4}}, \quad R_{2,4,2,4}=-\frac{3 a_{1} a_{3}-a_{2}^{2}}{4 a_{3}} .
\end{gathered}
$$

4. Непогружаемость $\mathrm{Nil}^{4}$ с произвольной левоинвариантной метрикой в $\mathbb{R}^{5}$. Допустим, что нилмногообразие $\mathrm{Nil}^{4}$ с левоинвариантной метрикой (3) погружено в $\mathbb{R}^{5}$. Тогда справедливы следующие уравнения Гаусса в единице $e$ (поскольку система переопределенная, мы вьписьваем только те из уравнений Гаусса, которые потребуются для доказательства ее противоречивости):

1) $R_{1,2,1,2}=b_{11} b_{22}-b_{12}^{2}=a_{1}^{2} /\left(4 a_{4}\right)$,

2) $R_{2,3,2,3}=b_{22} b_{33}-b_{23}^{2}=a_{2}^{2} /\left(4 a_{4}\right)$,

3) $R_{3,4,3,4}=b_{33} b_{44}-b_{34}^{2}=-3 a_{2} / 4$,

4) $R_{1,2,1,3}=b_{11} b_{23}-b_{13} b_{12}=0$,

5) $R_{2,3,2,4}=b_{22} b_{34}-b_{24} b_{23}=0$,

6) $R_{3,2,3,4}=b_{33} b_{24}-b_{32} b_{34}=0$,

7) $R_{1,3,1,4}=b_{11} b_{34}-b_{14} b_{13}=0$,

8) $R_{2,1,2,3}=b_{22} b_{13}-b_{23} b_{21}=a_{1} a_{2} /\left(4 a_{4}\right)$,

9) $R_{4,2,4,3}=b_{44} b_{23}-b_{42} b_{43}=0$,

10) $R_{1,2,3,4}=b_{13} b_{24}-b_{14} b_{23}=0$.

Действуя с тройкой уравнений 2), 5), 6) таким же образом, как при доказательстве теоремы 1 , получим, что $b_{34}=0$.

Оперируя затем так же с тройкой уравнений 3$), 6), 9)$ найдем, что $b_{23}=0$.

Тогда из уравнения 8) будет следовать, что $b_{13} \neq 0$.

Изучение уравнений 4), 7), 10) дает, что $b_{24}=b_{14}=b_{12}=0$.

Наконец, анализ тройки уравнений 1), 2) и 3) приводит к абсурду, что и доказьвает противоречивость выписанной системы уравнений Гаусса. 
5. Непогружаемость $\mathrm{Nil}^{3} \times \mathbb{R}$ в $\mathbb{R}^{5}$. Поскольку согласно [5, лемма 2.8] всякая левоинвариантная метрика на группе Гейзенберга $H_{1}$ изометрична некоторой левоинвариантной метрике с метрическим тензором в единице $е$ вида $\operatorname{diag}(a, a, 1), a>0$, то можно считать, что левоинвариантная метрика на $\mathrm{Nil}^{3} \times \mathbb{R}$ имеет вид

$$
d s^{2}=a\left(d x^{2}+d y^{2}\right)+(d z-x d y)^{2}+d t^{2},
$$

где $(x, y, z) \in \mathrm{Nil}^{3}, t \in \mathbb{R}$. Ненулевые компоненты тензора Римана данной метрики, вычисленные в Maple, следующие:

$$
R_{1,2,1,2}=-\frac{3 a-x^{2}}{4 a}, \quad R_{2,3,2,3}=R_{1,3,1,3}=\frac{1}{4 a}, \quad R_{1,2,1,3}=-\frac{x}{4 a} .
$$

Если предположить, что $\mathrm{Nil}^{3} \times \mathbb{R}$ допускает регулярное погружение в $\mathbb{R}^{5}$, то должны выполняться уравнения Гаусса. Рассматривая в точке $e$ тройку уравнений

1) $R_{1,2,2,3}=b_{12} b_{23}-b_{13} b_{22}=0$,

2) $R_{1,3,3,2}=b_{13} b_{32}-b_{12} b_{33}=0$,

3) $R_{2,3,2,3}=b_{22} b_{33}-b_{23}^{2}=1 /(4 a)$,

аналогично тому, как при доказательстве теоремы 1 , получим

$$
b_{13}=b_{12}=0
$$

Затем, рассматривая тройку уравнений

2) $R_{1,3,3,2}=b_{13} b_{32}-b_{12} b_{33}=0$,

4) $R_{2,1,1,3}=b_{21} b_{13}-b_{23} b_{11}=0$,

5) $R_{1,3,1,3}=b_{11} b_{33}-b_{13}^{2}=1 /(4 a)$,

получим, что $b_{23}=0$. Тогда тройка уравнений

3) $R_{2,3,2,3}=b_{22} b_{33}=1 /(4 a)$,

5) $R_{1,3,1,3}=b_{11} b_{33}=1 /(4 a)$,

6) $R_{1,2,1,2}=b_{11} b_{22}=-3 / 4$

противоречива, что и доказьвает непогружаемость $\mathrm{Nil}^{3} \times \mathbb{R}$ в $\mathbb{R}^{5}$.

\section{СПИСОК ЦИТИРОВАННОЙ ЛИТЕРАТУРЫ}

[1] Rivertz H. J. An obstruction to isometric immersion of the threedimensional Heisenberg group into $\mathbb{R}^{4}$ // Preprint series in Pure Mathematics № 22. Oslo: Matematick institut Univ. Oslo, 1999.

[2] Громов М. Дифференциальные соотношения с частными производными. М.: Мир, 1990.

[3] Hillman J. A. Four-manifolds, Geometries and Knots. Geometry and Topology Monographs. V. 5: Sydney, 2002.

[4] Кобаяси Ш., Номидзу К. Основы дифференциальной геометрии. Т. 2. М.: Наука, 1981.

[5] Gordon C. Riemannian manifolds isospectral on functions but not on 1-forms // J. Differential Geometry. 1986. V. 24. P. 79-96.

[6] Бессе А. Многообразия Эйнштейна. М.: Мир, 1990.

[7] Аминов Ю. А. Геометрия подмногообразий. Киев: Наукова думка, 2002.

Харьковский национальный университет им. В. Каразина, Украина

Поступило

E-mail: masaltsev@univer.kharkov.ua

25.09 .2003

Исправленный вариант

30.03.2004 